\title{
Forum COVID-19: Geistes- und sozialwissenschaftliche Perspektiven
}

Karen Nolte

Der Historiker Malte Thießen hat vor wenigen Jahren herausgestellt, dass sich gegenwärtige Gesellschaften im „Zeitalter der Immunität“ wähnten diese Gewissheit einer „Immunisierten Gesellschaft" hat nun durch COVID-19 Risse bekommen (Thießen 2017). Medizinhistorische Expertise ist derzeit zur Bewertung des gesellschaftlichen und politischen Umgangs mit der COVID-19-Pandemie in den populären Medien sehr gefragt. Vor COVID-19 folgte die öffentliche Darstellung und Wahrnehmung des klassischen medizinhistorischen Forschungsgebiets "Seuchengeschichte“ eher einem Fortschrittsnarrativ, demzufolge Menschen in vergangenen Gesellschaften gefährlichen Infektionskrankheiten schutzlos ausgeliefert waren, und die wiederkehrende Gefahr der Epi- und Pandemien dank des Siegeszugs der Bakteriologie im 20. Jahrhundert endgültig überwunden werden konnte. Nun wird der Auseinandersetzung mit historischen „Seuchen“, vor allem mit der Influenza-Pandemie von 1918-1920, der „Spanischen Grippe“, eine andere Funktion zugeschrieben: Aus der Geschichte sollen Politik und Gesellschaft Lehren für die aktuellen Herausforderungen der Pandemie ziehen. In dem Bewusstsein, selbst inmitten eines Pandemiegeschehens historischen Ausmaßes zu stehen, erhält die medizin- und wissenschaftsgeschichtliche Forschung über Epi- und Pandemien derzeit ebenso kräftigen Aufwind wie sozial- und kulturwissenschaftliche Analysen von Krisen und Katastrophen.

Für den Auftakt dieses Forums wurden daher Wissenschaftler*innen des skizzierten Forschungsfelds angefragt, ihre Expertise einzubringen und ihr Wissensgebiet vor dem Hintergrund von Beobachtungen des aktuellen gesellschaftlichen, politischen und wissenschaftlichen Umgangs mit der COVID-19-Pandemie in den letzten Wochen zu reflektieren sowie aktuelle Fragestellungen und Thesen zur Seuchen- bzw. Pandemiegeschichte herauszuarbeiten. Auch soll es darum gehen, jenseits der derzeitigen Leit- 
wissenschaft Virologie die gegenwärtige Situation einer wissenschaftlichen Analyse aus Sicht einer geistes- und sozialwissenschaftlich orientierten Wissenschaftsforschung zu unterziehen.

Mit den folgenden Beiträgen eröffnet NTM die wissenschaftliche Diskussion rund um COVID-19 und fordert dazu auf, weitere Beiträge aus Sicht von Medizin-, Wissenschafts- und Technikgeschichte und STS zum Thema (max. 15.000 Zeichen incl. Leerzeichen und Literatur) einzureichen.

Funding Open Access funding provided by Projekt DEAL.

Open Access Dieser Artikel wird unter der Creative Commons Namensnennung 4.0 International Lizenz veröffentlicht, welche die Nutzung, Vervielfältigung, Bearbeitung, Verbreitung und Wiedergabe in jeglichem Medium und Format erlaubt, sofern Sie den/die ursprünglichen Autor(en) und die Quelle ordnungsgemäß nennen, einen Link zur Creative Commons Lizenz beifügen und angeben, ob Änderungen vorgenommen wurden.

Die in diesem Artikel enthaltenen Bilder und sonstiges Drittmaterial unterliegen ebenfalls der genannten Creative Commons Lizenz, sofern sich aus der Abbildungslegende nichts anderes ergibt. Sofern das betreffende Material nicht unter der genannten Creative Commons Lizenz steht und die betreffende Handlung nicht nach gesetzlichen Vorschriften erlaubt ist, ist für die oben aufgeführten Weiterverwendungen des Materials die Einwilligung des jeweiligen Rechteinhabers einzuholen.

Weitere Details zur Lizenz entnehmen Sie bitte der Lizenzinformation auf http:// creativecommons.org/licenses/by/4.0/deed.de.

\section{Literatur}

Thießen, Malte (2017). Immunisierte Gesellschaft: Impfen in Deutschland im 19. und 20. Jahrhundert, Göttingen: V\&R.

Hinweis des Verlags Der Verlag bleibt in Hinblick auf geografische Zuordnungen und Gebietsbezeichnungen in veröffentlichten Karten und Institutsadressen neutral.

Karen Nolte

Institut für Geschichte und Ethik der Medizin

Ruprecht-Karls-Universität Heidelberg

Im Neuenheimer Feld 327

69120 Heidelberg

Deutschland

karen.nolte@histmed.uni-heidelberg.de 